\title{
Archives
}

\section{Théâtre à Montréal, 1825-1930 : un récit visuel mettant en scène des documents patrimoniaux}

\section{Danielle Léger}

Volume 48, numéro 1, 2019

$46^{\mathrm{e}}$ Congrès de l'Association des archivistes du Québec

URI : https://id.erudit.org/iderudit/1060816ar

DOI : https://doi.org/10.7202/1060816ar

Aller au sommaire du numéro

\section{Éditeur(s)}

Association des archivistes du Québec (AAQ)

ISSN

0044-9423 (imprimé)

2369-9256 (numérique)

Découvrir la revue

Citer cet article

Léger, D. (2019). Théâtre à Montréal, 1825-1930 : un récit visuel mettant en scène des documents patrimoniaux. Archives, 48(1), 89-106.

https://doi.org/10.7202/1060816ar
Résumé de l'article

L'auteur présente un projet de recherche-diffusion sur l'histoire culturelle, sociale et commerciale d'une quarantaine de lieux de théâtre montréalais actifs entre 1825 et 1930, réalisé en partenariat avec le Laboratoire d'histoire et de patrimoine de Montréal (LHPM) de l'UQAM. Puisant à la fois dans le patrimoine documentaire publié et les documents d'archives détenus par Bibliothèque et Archives nationales du Québec, ces travaux ont suscité la création de deux projets de diffusion complémentaires. Une collection a d'abord été créée au printemps 2017 dans Historypin, pour être ensuite déployée en près de deux cents images et capsules textuelles dans un parcours structuré porté par une autre plateforme Web, Story Maps. L’auteur commente ici la portée du projet, sa méthodologie et ses retombées documentaires. Les mérites respectifs des deux plateformes utilisées sont également mis en relief.
Ce document est protégé par la loi sur le droit d'auteur. L'utilisation des services d’Érudit (y compris la reproduction) est assujettie à sa politique d'utilisation que vous pouvez consulter en ligne.

https://apropos.erudit.org/fr/usagers/politique-dutilisation/ 


\section{EONGRÈS DE L'AAQ}

\section{Théâtre à Montréal, 1825-1930: un récit visuel mettant en scène des documents patrimoniaux}

\section{DANIELLE LÉGER}

Bibliothécaire responsable de collections, Bibliothèque et Archives nationales du Québec

Un individu lie la métropole montréalaise à l'assassinat d'Abraham Lincoln : il se nomme John Wilkes Booth. Ce comédien américain a joué au début des années 1860 sur la scène de la principale salle de spectacle montréalaise de l'époque, le Théâtre Royal de la rue Côté. Selon certains, un séjour au Saint Lawrence Hall en octobre 1864 aurait permis à Booth - plus discrètement cette fois - de rencontrer des militants pro-sudistes réfugiés à Montréal, en vue de l'attentat commis le 14 avril 1865 au Ford's Theatre de Washington.

D'une certaine manière, John Wilkes Booth est aussi l'étincelle qui a amené BAnQ à verser dans Story Maps un récit visuel sur I'histoire des lieux de théâtre à Montréal entre 1825 et 1930. En effet, le séduisant parcours cartographique sur l'assassinat d'Abraham Lincoln mis en ligne par Esri, le développeur de Story Maps, nous a d'emblée convaincus du potentiel de cet outil pour notre projet de diffusion sur le Web'. Avec la version gratuite de cette application développée en mode logiciel libre, il nous est apparu possible de produire un récit évocateur qui jumelle la géographie de Montréal, des textes narratifs et des images. 
Conçu dans l'esprit d'une contribution de BAnQ au 375e anniversaire de la fondation de Montréal, Théâtre à Montréal, 1825-1930 documente le rôle clé joué par la métropole montréalaise en tant que cité du spectacle. Cette ressource Web puise dans les collections patrimoniales et fonds d'archives de BAnQ. Elle se veut tous publics, avec le souhait que chacun puisse y trouver son compte, qu'il soit simple curieux, amateur d'images, créateur, étudiant ou spécialiste. Au cœur du projet: une quarantaine de lieux de théâtre et leur histoire depuis I'inauguration du premier Théâtre Royal (1825) jusqu'au tout début de la crise économique qui marquera les années 1930. L'approche retenue? Côté cour: un projet de recherche-diffusion sur l'histoire culturelle, sociale et commerciale du théâtre à Montréal. Côté jardin : un maillage étroit entre les ressources et expertises de BAnQ et celles des milieux universitaires. En lever de rideau: deux interfaces Web accessibles sans frais qui misent sur la localisation géographique.

\section{POURQUOI LE THÉÂTRE?}

Au Québec, le domaine théâtral repose sur trois groupes-clés: un réseau dynamique de créateurs-diffuseurs, une communauté active de chercheurs et un public enthousiaste. BAnQ côtoie activement ces milieux dans divers contextes. À titre d'exemples, des échanges fructueux se poursuivent avec des instances tels le Centre des auteurs dramatiques (CEAD), la Société québécoise d'études théâtrales (SQET), la Théâtrothèque du Centre de recherche interuniversitaire sur la littérature et la culture québécoises (CRILCQ), les Théâtres Associés inc. (TAI), I'École nationale de théâtre du Canada et la Maison Théâtre. L'instauration du dépôt légal des programmes de spectacles et son application, amorcée en 2004, interpellent diffuseurs et chercheurs. Des rencontres-découvertes autour des collections patrimoniales en théâtre et la diffusion numérique de ces dernières jettent de multiples passerelles entre les publics - amateurs ou spécialisés - et ce riche patrimoine.

Les arts du spectacle constituent un sujet porteur quand vient le temps de documenter l'histoire culturelle, sociale et commerciale d'une société. À la fois pôle de création et industrie culturelle, au croisement du répertoire et des avant-gardes, ce champ d'activité génère des corpus documentaires multiformes et fascinants dotés « d'une remarquable faculté d'adaptation à l'événement, au goût du jour» (La Gorce, 1996, p. 9). Pourtant, pour 
toutes sortes de raisons, ce pan du patrimoine d'intérêt québécois est encore largement inexploré, tant par les chercheurs que par les diffuseurs.

Des incursions du côté de la musique, de l'opéra, du cirque et du cinéma émaillent le récit visuel sur le théâtre créé par BAnQ. Au départ, nous souhaitions intégrer le théâtre musical, et tout particulièrement l'opéra. Bien vite, dès le début des travaux de recherche, nous avons réalisé que nous ne pouvions parler de la salle de l'Orpheum sans mentionner les récitals organisés par l'impresario Joseph-Albert Gauvin; ni passer sous silence les spectacles de cirque au Jardin Guilbault (Figure 1); ni ignorer les liens, empreints de complicités ou de rivalités, qui se tissent entre théâtre et cinéma à compter des années 1890 . Nous ne pouvions non plus laisser dans l'ombre la place de la chanson au théâtre. Nous avons donc délibérément choisi de témoigner des métissages entre les multiples arts du spectacle qui marquent la destinée des lieux de théâtre à Montréal.

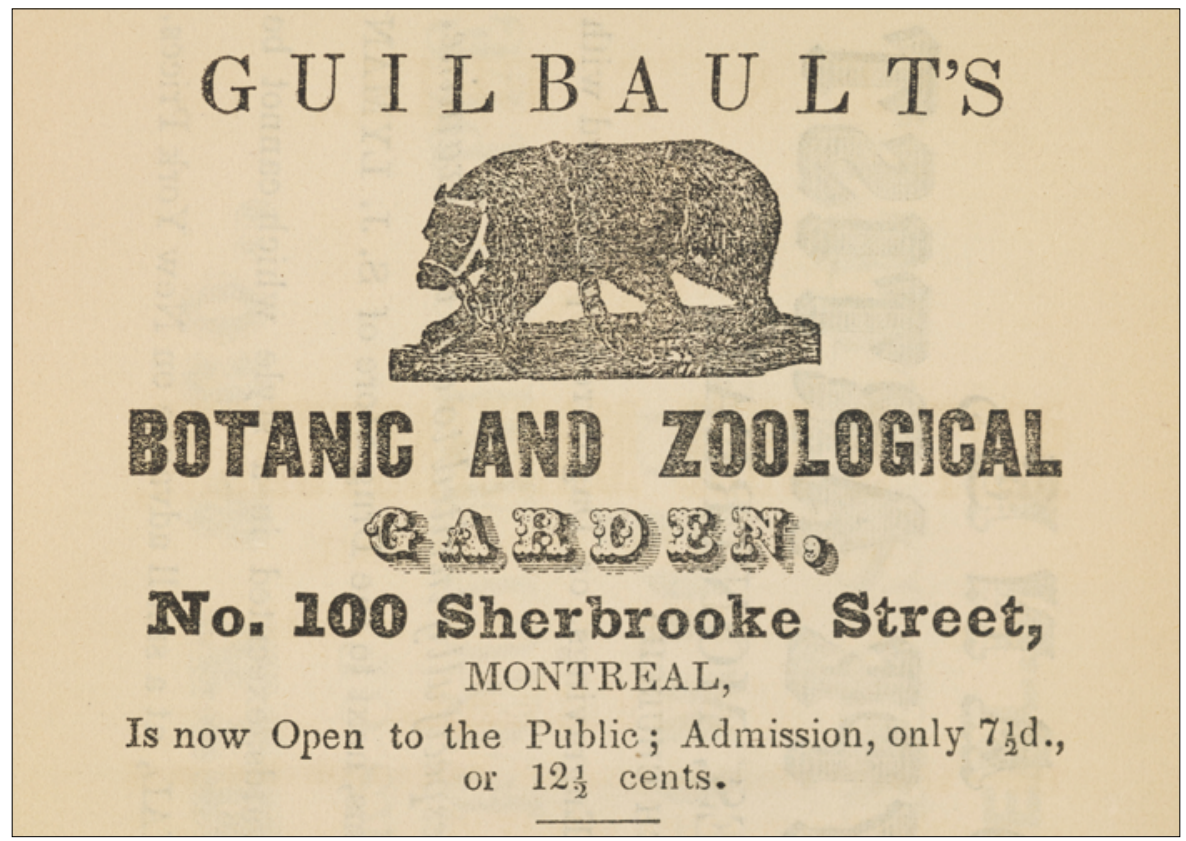

Figure 1: Publicité tirée du livre de MACKAY, R. W. S. (1854). The Stranger's guide to the cities and principal towns of Canada. Montreal: C. Bryson, p. 139. Source: BAnQ, Collection nationale, 917.14042 S8975 1854. 
Pourquoi se confiner à 105 ans d'histoire? Par réalisme d'abord, compte tenu du foisonnement de la création théâtrale plus récente. Également en raison du droit d'auteur, puisqu'il nous fallait limiter les démarches d'obtention des droits pour les documents qui ne sont pas du domaine public. La fondation du Théâtre Royal-Molson en 1825 - considéré comme la première salle de spectacle permanente à Montréal - s'est vite imposée en début de période. Pour faire un beau siècle tout rond, I'année 1925 avait d'abord été retenue comme date butoir. Toutefois, au fil des travaux, une balise plus naturelle, plus significative s'est imposée avec l'année 1930 puisqu'elle marque le début de la Crise économique et l'arrivée de la radio qui va bouleverser significativement les habitudes culturelles du public et la vie des théâtres montréalais. Compte tenu des droits déjà négociés par BAnQ, le projet a suscité des démarches auprès de quelques titulaires de droit (notamment le Théâtre du Nouveau Monde, les Archives des Jésuites au Canada et le YIVO Institute for Jewish Research de New York).

\section{UN PROJET DE RECHERCHE-DIFFUSION}

Investie d'une mission évoquant par moments celle de l'archéologue, l'équipe du projet Théâtre à Montréal, 1825-1930 a mis au jour plusieurs sources méconnues ou oubliées, certaines affectées par l'absence de numérisation ou un déficit de signalement. Un esprit de convergence entre archives, bibliothèques et musées a également balisé le projet. Les documents sont ici traités à la fois comme des illustrations, des sources et des artefacts. Ces manifestations de la culture matérielle assument la part visuelle du récit, alimentent l'interprétation historique (véhiculée à son tour par les commentaires écrits qui accompagnent chacune des illustrations) et agissent comme des artefacts, témoins directs d'une époque révolue.

Finalement, les trois quarts des quelque deux cents documents retenus proviennent de neuf des collections d'imprimés publiés détenus par BAnQ; une cinquantaine provient de ses fonds d'archives. Avec le quart des visuels, l'ensemble documentaire qui domine cette sélection est sans conteste la collection patrimoniale de programmes de spectacles. Toutefois, avec près de cent vingt images, les documents iconographiques (affiches, cartes postales, illustrations et photographies) comptent pour près de $60 \%$ de l'ensemble total. En écho à la perspective sociale et commerciale adoptée, nous avons pris soin d'inclure des caricatures et du matériel publicitaire. 
La numérisation récente en mode plein texte de plusieurs revues, journaux et programmes de spectacles propulse la recherche vers des résultats inespérés, parfois inédits. Dans les coulisses de ce projet d'histoire appliquée, plusieurs autres documents patrimoniaux ont appuyé nos recherches. Les annuaires Lovell et les plans d'assurance incendie ont notamment permis une géolocalisation aussi précise que possible des lieux de théâtre et de certains sites voisins étroitement rattachés à la vie des théâtres et à l'expérience des spectateurs (restaurants, hôtels, commerces, etc.).

Le récit visuel proposé ouvre une fenêtre sur une histoire mouvementée, ponctuée d'initiatives parfois éphémères (Théâtre des Variétés, Théâtre de la Renaissance, Café-concert Eldorado). On y croise des salles prestigieuses (Academy of Music, His/Her Majesty's Theatre), des salles populaires (Théâtre Royal-Côté), des lieux de divertissement polyvalents (Crystal Palace, Jardin Guilbault, Parc Sohmer), des salles institutionnelles (Mechanics' Hall, Gesù), des lieux culturels emblématiques (MonumentNational, Théâtre National). Plusieurs de ces édifices ont disparu, certains ont toujours pignon sur rue et d'autres ont conservé leur vocation initiale, tel le Théâtre Saint-Denis.

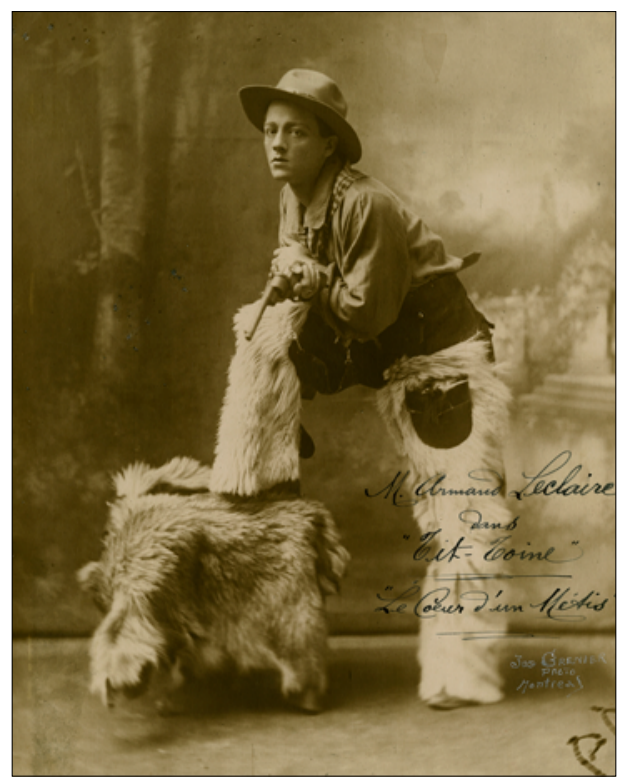

Figure 2: Armand Leclaire incarnant Tit-Toine dans Le Cœur d'un Métis. Photographie de Jos Grenier photo, Montréal, vers 1920.

Source: BAnQ Vieux-Montréal, Fonds Fred Barry et Bella Ouellette, MSS446,S3,SS1,D2,P1. 
On a délibérément choisi de parler de célébrités comme la cantatrice d'origine québécoise Emma Albani, la grande tragédienne française Sarah Bernhardt, qui a visité Montréal à neuf reprises, et le réputé illusionniste Harry Houdini, dont le décès n'est certes pas sans lien avec son escale dans la métropole en 1926, mais aussi de figures moins connues du grand public, tels John Bolingbroke Sparrow, Paul Cazeneuve, Georges Gauvreau et Armand Leclaire (Figure 2). II y est aussi question des pratiques sociales, commerciales et culturelles entourant l'activité des théâtres, du théâtre yiddish aux chapeaux des spectatrices, sans oublier le contrôle new-yorkais sur les salles montréalaises. La présence des spectateurs et des gens de théâtre dans les restaurants et les hôtels de la métropole est l'objet de quelques capsules.

\section{UN RICHE PATRIMOINE ICONOGRAPHIQUE}

Le recours aux images instaure un rapport à la fois affectif et cognitif où se conjuguent le plaisir immédiat de la perception et l'acquisition de connaissances. Bien implantée en milieu muséal, cette approche gagne actuellement en faveur dans le champ des sciences humaines et dans les approches didactiques préconisées en milieu scolaire, notamment pour l'apprentissage de I'histoire. Les documents iconographiques gagnent peu à peu leurs lettres de noblesse en tant que sources historiques à part entière.

Dans le domaine des études théâtrales, les chercheurs ont longtemps privilégié les sources textuelles, préconisant une histoire littéraire, tournée vers les textes dramatiques. La prise de conscience de l'intérêt des sources iconographiques pour la recherche et l'enseignement en théâtre aurait commencé à émerger vers la fin des années $1950^{2}$. Plus récemment, l'étude des imprimés éphémères (publicités, affiches, programmes de spectacles, etc.) semble emprunter une évolution analogue (Figure 3). "L'histoire est changeante, les images sont multiples, mais I'observation des liens qui les unissent est féconde », écrit Annie Duprat (2007, p. 6). Tous les spécialistes émettent toutefois la même mise en garde: au moment d'utiliser les sources iconographiques, il faut savoir les décoder en exerçant son esprit critique. II faut les observer attentivement, analyser leur matérialité et leurs conditions de production «tout en les replaçant dans le contexte de la société qui les a produites» (Duprat, 2007, p. 4). 
I| s'agit, à chaque nouvelle image, de mesurer la «valeur de vérité » de cette trace du passé en fonction du point de vue qu'elle exprime (Larouche, 2014, p. 214).

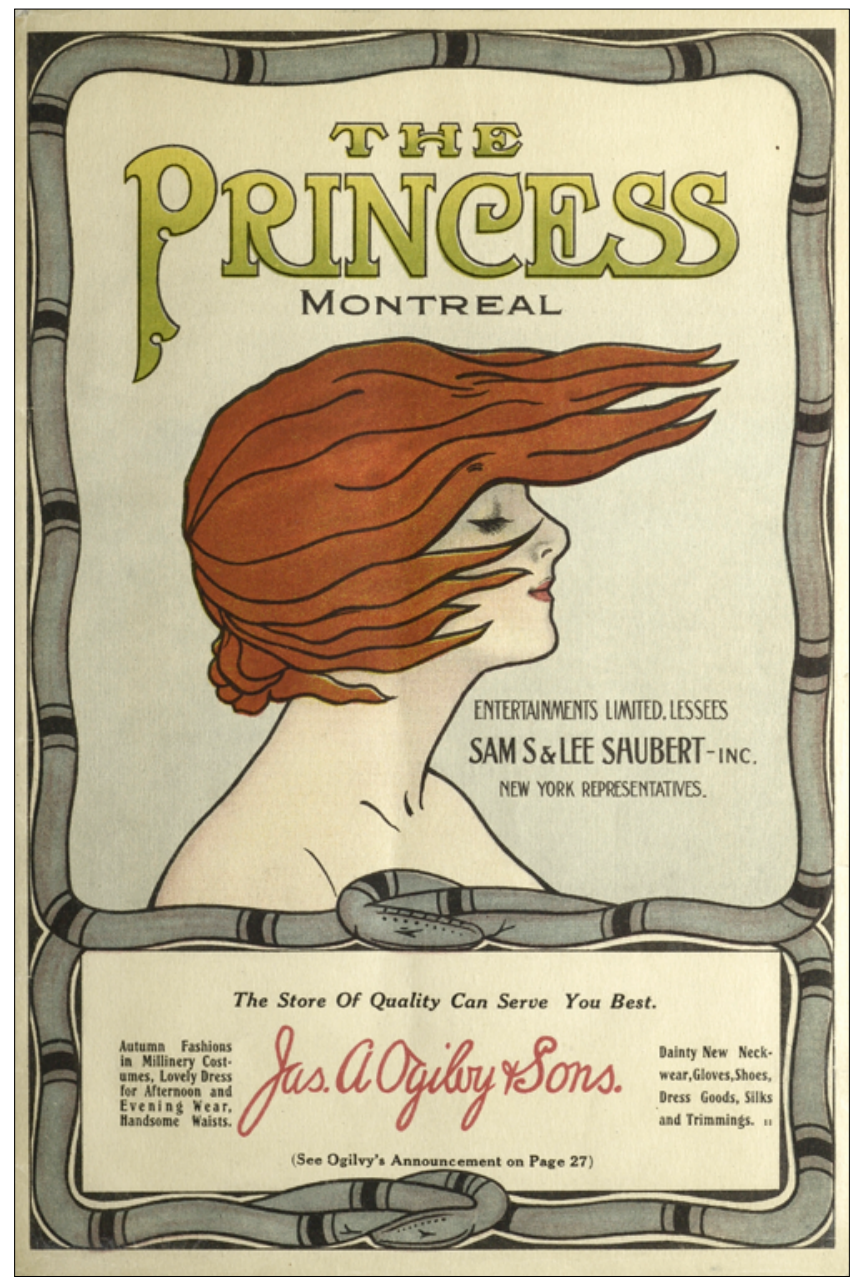

Figure 3: Couverture du programme du spectacle Mr. Veitch presents the renowned operatic soprano Madame Nordica, Montréal, The Princess Theatre, 1912. Source: BAnQ Rosemont-La Petite-Patrie, PRO PRITHE 1912.10.07 x.

Voici trois exemples de photographies tirées des archives de BAnQ qui ont comblé notre souci d'évoquer l'environnement et la vie des lieux de 
théâtre. Les deux premières proviennent des incontournables albums d'images constitués par Édouard-Zotique Massicotte ${ }^{3}$ et documentent I'apparence du site actuellement occupé par le Palais des Congrès au tournant du $X X^{e}$ siècle. La photographie retenue pour illustrer le Théâtre Royal-Côté témoigne avec éloquence des lignes électriques, de la présence du tramway et des grandes affiches illustrées collées sur une palissade de ce site complètement transformé au fil du temps. Selon nos recherches, l'image daterait de la fin des années 1890. Le deuxième cliché, capté vers 1910, permet d'illustrer les pratiques d'affichage publicitaire de l'époque. Sous les réclames d'un magasin de vêtements pour dames et des fabricants du savon Baby's Own, des affiches annoncent la comédie romantique Monsieur Beaucaire, au Princess Theatre de la rue Sainte-Catherine, en tournée depuis Broadway. La troisième photographie, signée par le photoreporter Conrad Poirier, date de 1940. Elle a été croquée devant le MonumentNational. On y aperçoit l'entrée principale qui donne accès à sa grande salle de spectacle, mais aussi un de ses locataires, le très flamboyant cinéma Starland et - témoignage parlant de la vie sur la Main - un attroupement de parieurs clandestins (Figure 4).

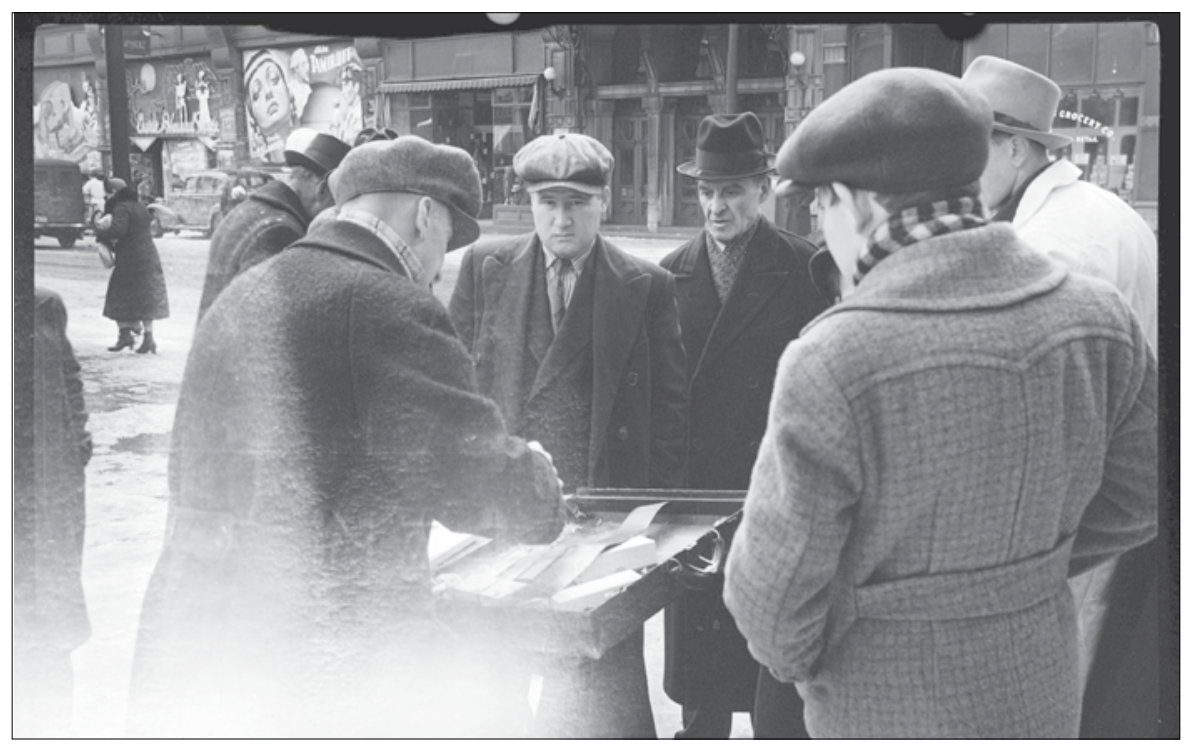

Figure 4: Conrad Poirier, Feature: Main Street, photographie, 16 mars 1940. Source: Fonds Conrad Poirier, BAnQ Vieux-Montréal, P48,S1,P5207. 
Petite ombre au tableau: le repérage dans les collections de BAnQ a laissé neuf lieux de théâtre sans représentation de leur façade ou de leurs espaces intérieurs. C'est le cas du Théâtre Royal-Olympic, du Jardin Guilbault, du Théâtre de la Renaissance, du Café-concert Eldorado, du Palais-Royal, du Théâtre Delville, du Théâtre de la Gaieté Française, du Théâtre Canadien-Français et du Théâtre Chanteclerc. Bien qu'étendu sur une période de trois ans, notre calendrier de production était serré. II ne nous a pas permis de puiser dans d'autres collections institutionnelles pour combler ces lacunes; il a également court-circuité notre projet d'exploiter davantage les documents cartographiques d'époque. Notre objectif de mise en valeur des fonds et collections de BAnQ nous semble atteint. Souhaitera-t-on multiplier les capsules? Ajouter de nouveaux lieux de théâtre? Couvrir d'autres villes? La suite du projet reste ouverte.

\section{UN MAILLAGE FRUCTUEUX}

Le souhait de créer une ressource cartographiant les lieux de théâtre à Montréal était en germe depuis quelques années. II s'est matérialisé dans des conditions favorables. Lorsque BAnQ a investi la plateforme collaborative Historypin à compter de 2014, les moyens techniques étaient réunis pour décrire, dater, commenter et géolocaliser des images. Au cours des premières années d'utilisation, BAnQ y a surtout versé des cartes postales et des photographies représentant des éléments du paysage urbain.

Le Laboratoire d'histoire et de patrimoine de Montréal (LHPM) de I'Université du Québec à Montréal ${ }^{4} \mathrm{~s}^{\prime}$ est avéré un véritable tremplin pour ce chantier de recherche et de rédaction. Le partenariat de recherche Montréal, plaque tournante des échanges: histoire, patrimoine, devenir a ainsi fourni un contexte inespéré pour aborder un objet de recherche lui-même marqué par la circulation des œuvres et des comédiens, par le caractère éphémère des lieux, des manifestations et des artefacts imprimés, par les multiples métissages culturels et disciplinaires. Entre autres retombées, nous avons pu compter sur le soutien logistique et la collaboration scientifique de Joanne Burgess, historienne et directrice du LHPM, ainsi que sur le travail des adjointes de recherche recrutées par le Laboratoire, Éliane Bélec et Jacinthe Blanchard-Pilon, qui ont consacré plus de sept cents heures au projet. Nous avons aussi bénéficié des conseils 
avisés de Léon Robichaud, du département d'histoire de l'Université de Sherbrooke et codirecteur du LHPM, et de la rétroaction des membres du laboratoire.

L'alliance avec le milieu universitaire s'est aussi manifestée du côté des programmes de stage en sciences de l'information de l'Université de Montréal et de I'Université McGill. Cette contribution s'est avérée essentielle, en amont comme en fin de projet. Au total, quatre stagiaires ont été mis à contribution pour la recherche préliminaire d'artefacts dans les collections de BAnQ et la mise en ligne des contenus dans Historypin.

Amorcé au printemps 2015, le projet s'est déployé en cinq phases, entrecoupées de pauses, soit pour des raisons logistiques, soit parce que le quotidien de la bibliothèque nous ramenait vers les demandes de référence, les propositions d'acquisition, le suivi de la numérisation et autres projets.

Phase 1: La phase initiale de repérage dans les fonds et collections, menée en parallèle par trois stagiaires en sciences de l'information, a permis d'identifier en quelques semaines environ quatre cent trente artefacts issus de huit familles documentaires.

Phases 2 et 3: Sur une période de trois ans, avec le concours actif et compétent des deux adjointes de recherche embauchées par le LHPM ${ }^{5}$, nous avons mis en place les outils et le cadre méthodologique, puis développé les contenus.

Sur le plan méthodologique, nous avons:

- décliné les facettes utiles au développement des contenus: lieux de théâtre, créateurs et artisans, troupes, œuvres, champs artistiques et genres, contexte des spectacles;

- défini une structure à deux niveaux: une ou deux capsules principales résumant I'histoire de chacun des lieux de théâtre, assorties au besoin de capsules secondaires explorant un aspect du lieu ou abordant les autres facettes;

- développé une bibliographie de sources secondaires sur l'histoire du théâtre, classées par période et par zone géographique, avec leur cote ou adresse Web (qui s'avérera aussi utile pour le travail de référence et de mise en valeur des collections qui se fait à $B A n Q$ ); 
- adopté la version gratuite de l'outil Web collaboratif COGGLE qui permet de créer en ligne d'efficaces «représentations mentales» (mind maps) qui nous ont permis d'organiser et de faire évoluer nos idées et notre plan de travail ${ }^{6}$.

Chacune des capsules a été rédigée selon un canevas comportant cinq éléments: un titre, un texte de longueur variable dépassant rarement les deux cents mots, la localisation du théâtre, une légende décrivant l'artefact choisi et l'adresse de l'artefact sur le portail de BAnQ. Certains lieux de théâtre n'ont donné lieu qu'à une simple capsule, faute de renseignements ou d'artefacts disponibles. Le recordman toutes catégories est le fameux Théâtre National de la rue SainteCatherine avec deux capsules principales et pas moins de vingt capsules secondaires!

Phase 4: Après la validation finale des contenus, la mise en ligne a suivi avec le concours d'une quatrième stagiaire en bibliothéconomie (volet Historypin), puis d'une professionnelle engagée par BAnQ (volet Story Maps).

Phase 5: La validation scientifique par des spécialistes de l'histoire du théâtre - initialement prévue avant la mise en ligne - est reportée au printemps 2018.

\section{SUR LE WEB, AVEC HISTORYPIN}

Historypin est une plateforme Web développée en partenariat avec Google. Issu de la Shift Foundation (une entreprise d'économie sociale auparavant connue sous le nom We are what we do), le développeur de cette ressource est un organisme sans but lucratif basé à Londres, à San Francisco, à La Nouvelle-Orléans, à Sofia et au Nouveau-Mexique. Parvenu à sa septième version, I'outil offre aux organismes à vocation culturelle la possibilité de « partager des photos et des histoires, racontant leur histoire locale ${ }^{7}$. II permet de repérer des sources iconographiques et d'explorer I'histoire par images, par mots-clés ou en naviguant sur Google Maps. Il est aussi possible de superposer l'image à une représentation récente du paysage urbain en mode Street View: une glissière active des effets en fondu enchaîné où dialoguent passé et présent (Figure 5). II s'agit $d^{\prime}$ 'un environnement collaboratif, les internautes pouvant interagir avec 
les contenus mis en ligne en les commentant ou en développant leurs propres histoires.

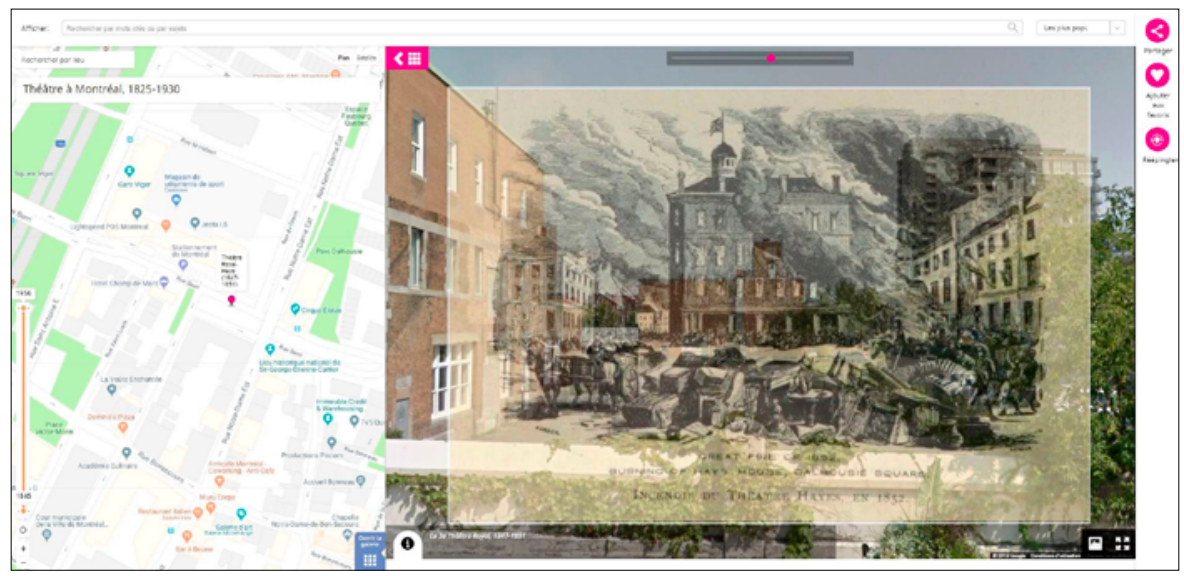

Figure 5: Dans Historypin, une image de l'édifice du troisième Théâtre Royal (Hayes), de 1852, superposée à une vue contemporaine du site en mode Google Street View.

Depuis avril 2017, les capsules principales de lieux de Théâtre à Montréal, 1825-1930 constituent une petite collection dans Historypin. Cet ensemble côtoie ici d'autres collections et parcours regroupant un millier de documents visuels d'intérêt québécois versés à ce jour par $\mathrm{BAnQ}^{8}$. Quatre autres collections, principalement constituées de cartes postales documentant les gares, les épiceries, les commerces et les sites industriels de Montréal, sont également issues du partenariat avec le LHPM. Comme pour la collection sur le théâtre, qui aborde indifféremment les théâtres huppés et les lieux de divertissement populaire, la documentation visuelle de l'histoire de Montréal met ici en relief des lieux fréquentés par des gens de toutes les classes sociales: gares monumentales (Bonaventure, Windsor, Viger) et gares de quartiers ouvriers, grands magasins (Henry Morgan, Eaton, Dupuis Frères), commerces à bon marché et petites épiceries de quartier, lieux de production industrielle où œuvrent patrons et travailleurs.

En dépit de ses nombreux atouts, Historypin intégrerait difficilement les nombreuses capsules secondaires sur les théâtres générées par ce projet. II s'est avéré impossible d'y accommoder efficacement un contenu structuré selon deux niveaux; il nous paraissait aussi peu 
heureux d'épingler un nombre relativement important d'éléments sur certains points de la carte. II fallait trouver une autre plateforme, flexible et attrayante. En ces temps d'austérité, nous devions opter pour une solution peu coûteuse et aussi ouverte que possible.

On a d'abord dessiné une maquette idéale pour préciser nos souhaits, soit une sorte de croisement entre un répertoire systématique et une exposition virtuelle. Nous avons considéré quatre solutions:

- le logiciel de gestion de bibliothèque numérique Omeka, mis au point par le Center for History and New Media (CHNM) de I'université George Mason;

- I'initiative Google Arts \& Culture qui met des milliers d'œuvres d'art à la portée du public;

- la solution WordPress qui permet de créer rapidement un site Web en choisissant parmi près de trois cent cinquante thèmes;

- I'application Story Maps qui a suscité notre adhésion immédiate... avec la complicité de John Wilkes Booth.

\section{SUR LE WEB, AVEC STORY MAPS}

Story Maps permet d'élaborer des « récits géographiques » en associant cartes géographiques, texte narratif, images et ressources multimédias. Basé à Toronto, Esri Canada est le créateur de Story Maps. ArcGIS, le logiciel derrière cette application, est un SIG (système d'information géographique) basé sur des technologies Web et créé en 1999. II est utilisé par diverses organisations des secteurs privé et public pour relier cartes, applications, données et personnes. C'est un puissant outil de collecte, de gestion de données, de référence, d'analyse, de partage et d'aide à la décision. Dans le secteur du patrimoine culturel, on l'utilise notamment pour cataloguer et gérer des ressources archéologiques et architecturales.

Pour le projet sur le théâtre à Montréal, j'ai travaillé avec ma collègue bibliothécaire Arianne Parent-Touchette qui a procédé à la mise en ligne des contenus tout en explorant les possibilités de ce nouvel outil. BAnQ a bénéficié du soutien ponctuel de deux équipes d'Esri, celle de Toronto et celle de Montréal. Nous avons utilisé la version gratuite, sans y associer 
de données statistiques, en misant sur le potentiel éducatif, visuel et narratif de Story Maps. La carte géographique agit ici comme un pôle d'ancrage pour des textes d'intérêt culturel et historique. Le récit est automatiquement hébergé sur ArcGIS Online. Quant aux images, elles ont été déposées sur Flickr.

La page d'accueil du site a été formatée selon le modèle Story Map Cascade. Bien que doté d'un séduisant "défilement immersif plein écran », ce modèle n'était peut-être pas la meilleure option, ne serait-ce que par son incompatibilité avec les navigateurs moins récents. Pour cette raison, nous utilisons généralement l'adresse Web qui donne directement accès aux capsules de premier niveau, sans passer par cette page d'accueil. Pour le reste des contenus, nous avons adopté le modèle Story Map Tour qui propose une visite où chaque élément du récit est géolocalisé. L'utilisateur peut emprunter un parcours séquentiel en cliquant dans la narration. II peut aussi naviguer librement soit en parcourant la carte interactive, soit en utilisant la frise de miniatures qui se déploie au bas de l'écran.

Quelques ajustements salutaires ont été apportés au récit visuel après son lancement public le 31 mai 2017 au congrès annuel de I'Association des archivistes du Québec. Nous avons découvert que Story Maps n'est pas conçu pour accommoder aisément plus d'un niveau d'information. Le premier niveau a été épuré pour en faire une page-pivot offrant un panorama général et un outil de repérage plus performant. Ce parcours initial (map tour) donne accès à chacun des parcours consacrés à un lieu spécifique. Un hyperlien appelé Pour en savoir plus sur ce lieu permet d'accéder à ce deuxième niveau de contenu (Figure 6). La fluidité de la navigation reste imparfaite: cela ne fonctionne que dans un sens. Il faut sélectionner manuellement l'onglet initial pour revenir au parcours d'origine (espérons que cette manœuvre sera intuitive pour les utilisateurs). Autre avantage offert par cette structuration des contenus: l'intégralité des contenus associés à un lieu - capsules principales et secondaires comprises - peut être citée par une adresse URL unique, devenant accessible en un seul clic $^{9}$. 


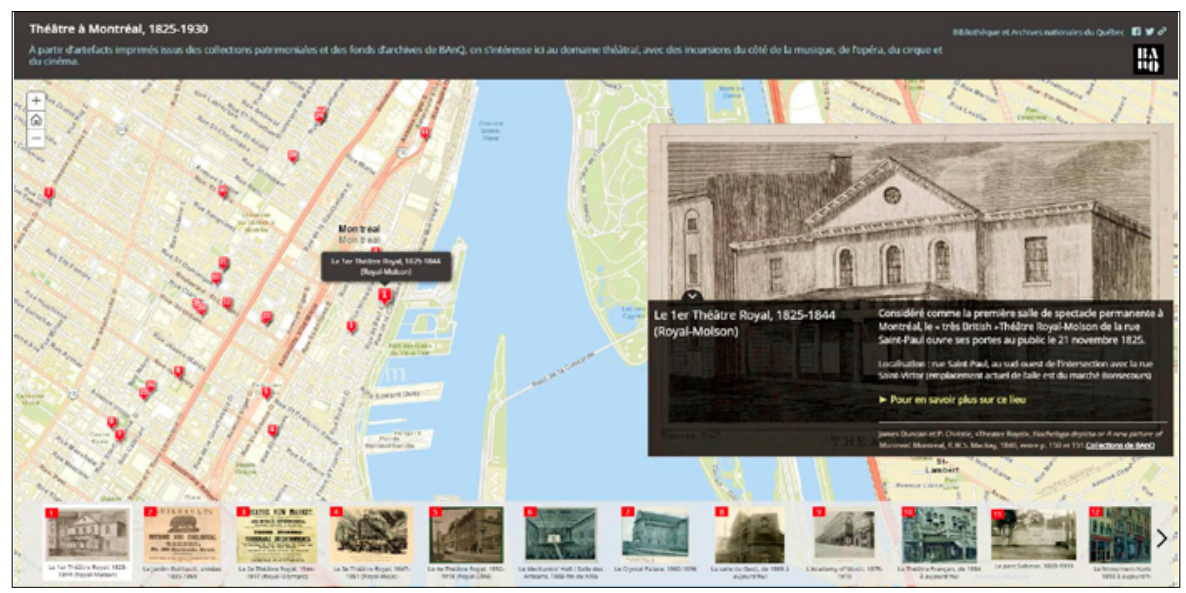

Figure 6: Dans Story Maps, la page-pivot qui permet de localiser les lieux de théâtre et qui donne accès aux parcours spécifiques.

Avant de procéder au versement dans Story Maps (c'est le cas aussi avec Historypin), il est avisé de finaliser ses contenus textuels et de les préparer avec soin, incluant les quelques balises HTML de base requises pour la mise en forme. Ces interfaces sont assez peu amicales quand vient le temps de retoucher les textes, surtout si ceux-ci sont substantiels.

Après quelques tests, nous avons dû ajouter des bandes latérales noires aux illustrations verticales afin de permettre au texte d'accompagnement de se déployer correctement. La version gratuite de Story Maps impose une limite de chargement de 85 images à la fois, ce qui nous a amenés à tout reprendre en segmentant notre album Flickr. La présence des images retravaillées avec des bandes latérales noires et des miniatures étonnera sans doute certains internautes.

Les utilisateurs de l'application derrière Story Maps sont autorisés à la modifier en téléchargeant le code source. Esri incite d'ailleurs la communauté des utilisateurs-développeurs à déposer les versions modifiées sur GitHub, un service Web d'hébergement et de gestion de développement de logiciels. Toutefois, contrairement à Historypin, Story Maps n'est pas conçu d'emblée comme une interface collaborative pour les utilisateurs externes. 


\section{CONCLUSION}

Malgré quelques bémols, les deux plateformes utilisées se sont avérées inspirantes. II faut saluer ici la collaboration indéfectible et précieuse des équipes de développement d'Historypin et de Story Maps. L'expérience est concluante à plusieurs égards:

- accessibilité accrue et mise en valeur du patrimoine documentaire à peu de frais;

- diffusion de connaissances historiques rigoureusement validées grâce au partenariat BAnQ/LHPM;

- métadonnées enrichies pour le catalogue de BAnQ (notamment pour les images contenues dans les albums d'Édouard-Zotique Massicotte);

- nouveaux artefacts numérisés;

- nouveau matériel disponible pour d'autres projets de diffusion.

Les métadonnées géographiques générées vont permettre, à terme, la recherche et le repérage par géolocalisation dans BAnQ numérique, la nouvelle interface regroupant les ressources numériques de l'institution.

Dans un tel projet, une fonction clé des organismes documentaires se démarque pleinement, soit une "garantie d'authenticité relevant [d'un] continuum patrimonial » (Larouche, 2014, p. 226), décuplée par la capacité d'interprétation des chercheurs universitaires. Et maintenant, pourquoi ne pas rêver d'un outil de valorisation qui tablerait à la fois sur le potentiel narratif et graphique de Story Maps et sur l'intégration de Google Maps et Google Street View que permet Historypin?

DANIELLe LÉGer 


\section{NOTES DE FIN}

1. Ce parcours, intitulé The Assassination of Abraham Lincoln, peut être consulté à I'adresse suivante: http://arcg.is/1poYIQb.

2. À ce sujet, on consultera le texte de Robert Erenstein cité en bibliographie.

3. http://numerique.banq.qc.ca/ressources/details/ezmas

4. Pour plus de renseignements sur le LHPM et ses travaux: https://hpm.uqam.ca/ le-laboratoire/a-propos/.

5. Il s'agit d'Éliane Bélec, historienne, et de Jacinthe Blanchard-Pilon, historienne de I'art, toutes deux diplômées de I'UQAM. Les principaux collaborateurs au projet sont identifiés sur le site du LHPM: https:///hpm.uqam.ca/programmation-scientifique/ patrimonialisation-commemoration-et-exposition/theatre-montreal-1825-1930/

6. Autour d'une idée centrale, COGGLE permet de créer une arborescence dont les éléments peuvent être déplacés, annotés et illustrés par des images. On crée un compte en quelques secondes. Depuis l'application, on peut inviter une ou plusieurs personnes à les lire, les éditer et/ou les commenter. La version gratuite permet aussi de les exporter au format PDF ou PNG. https://coggle.it/?lang=fr

7. Ce slogan figure en page d'accueil du site d'Historypin. https://www.historypin.org/fr/

8. L'adresse http://bit.ly/2k86k6v donne accès à l'ensemble des collections et parcours créés par BAnQ dans Historypin.

9. Pour obtenir l'adresse brève d'un parcours, il suffit de cliquer sur l'icône du maillon de chaîne $c$ qui se trouve en haut à droite de la page Web. Les capsules singulières d'un parcours ne peuvent faire l'objet d'un hyperlien spécifique.

\section{BIBLIOGRAPHIE}

BIBLIOTHĖQUE ET ARCHIVES NATIONALES DU QUÉBEC. (2017). Théâtre à Montréal, 1825-1930. Repéré en version abrégée dans Historypin à http://bit.ly/2k86k6v. Repéré en version intégrale dans Story Maps à http ://arcg.is/2nADeyV. Accès direct aux capsules de lieux repéré dans Story Maps à http ://arcg.is/2kWSC7d.

DUPRAT, A. (2007). Images et histoire: outils et méthodes d'analyse des documents iconographiques. Paris: Belin. 
ERENSTEIN, R. (1999). Theatre iconography: tradition, techniques, and trends. Dans Heck, T. et al. Picturing performance: the iconography of the performing arts in concept and practice (p. 136-149). Rochester, NY: University of Rochester Press.

LA GORCE, J. de. (1996). Introduction. Dans /conographie et arts du spectacle: actes du séminaire CNRS (p. 9). Paris: Klincksieck.

LAROUCHE, M.-C. (2014). Voir et savoir interpréter des documents iconographiques, de l'affectif au cognitif. Dans Éthier, M.-A., Lefrançois, D. et Demers, S. (dir.), Faire aimer et apprendre l'histoire et la géographie au primaire et au secondaire (p. 213-231). Québec: MultiMondes. 